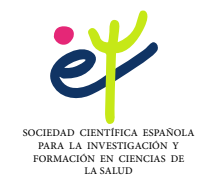

European Journal of Health Research

2021, Vol. 7, No 1 (Págs. 1-13)

Eur. J. Health. Research. e-ISSN 2445-0308

https://revistas.uautonoma.cl/index.php/ejhr doi: $10.32457 /$ ejhr.v7i1.1396

\title{
Tensiones y realidades de los docentes universitarios frente a la pandemia Covid-19
}

\author{
Tensions and realities of university teachers in the face \\ of the Covid-19 pandemic
}

\author{
Gladys Galvis* (D), Andrea Vásquez (D), Yaneth Patricia Caviativa (D), \\ Paola Andrea Ospina (D), Verónica Tatiana Chaves (D), Lina María Carreño (D), \\ y Víctor Javier Vera (D) \\ Universidad Manuela Beltrán (Colombia)
}

\section{RESUMEN}

La contingencia generada a partir de la pandemia de Covid-19 hace que las condiciones laborales de los docentes se transformen con un aumento de las horas laboradas, inmovilidad en el lugar de trabajo, resolución de situaciones administrativas de la institución educativa y el apoyo a estudiantes con situaciones que así lo requieren, lleva a una sobrecarga física y mental por parte de los docentes universitarios. Se desarrolla un estudio de diseńo fenomenológico en el que se incluyen 178 docentes universitarios del ciclo 2020-I con contrato laboral medio tiempo (4 horas) en horario de la mañana- noche y contrato laboral tiempo completo ( 8 horas); que se encuentran laborando desde sus casas en metodología teórica, práctica y teórico-práctica. Se encuentra que los docentes evidencian afectación en su carga física y mental debido a la contingencia por pandemia Covid-19, relacionando la jornada laboral, intensidad de trabajo, las relaciones interpersonales con respecto a los diferentes tipos de cargas desde la habitualidad al contexto especial dado por la contingencia, en donde el esfuerzo físico-mental, las situaciones de tipo estresantes en la actividad laboral y extra laboral son factores atribuidos al desgaste profesional del docente, la modalidad virtual ha generado que los docentes tengan conductas físicas y psicológicas cambiantes.

Palabras clave: Carga laboral, docentes universitarios, carga mental.

\footnotetext{
* Correspondencia: Gladys Galvis López. Vicerrectoría de Investigaciones. Universidad Manuela Beltrán. Carrera 1, no 60-00. Bogotá (Colombia). E-mail: gladys.galvis@docentes.umb.edu.co
} 


\begin{abstract}
The contingency generated from the Covid-19 pandemic means that the working conditions of teachers are transformed with an increase in the hours worked, immobility of the workplace, resolution of administrative situations of the educational institution and support for students with Situations that require it, leads to a physical and mental overload on the part of university teachers. A phenomenological design study is developed in which 178 university teachers of the 2020-I cycle are included with a part-time employment contract ( 4 hours) in the morning-night hours and a full-time employment contract ( 8 hours); who are working from their homes in theoretical, practical and theoretical-practical methodology. It is found that teachers show impairment in their physical and mental load due to the Covid-19 pandemic contingency, relating the working day, work intensity, interpersonal relationships with respect to the different types of loads from habituality to the given special context Due to contingency, where physical-mental effort, stressful situations in work and nonwork activities are factors attributed to the professional burnout of the teacher, the virtual modality has generated that teachers have changing physical and psychological behaviors.
\end{abstract}

Keywords: Workload, university teachers, mental load.

Jaspers dijo: "Soy en la medida en que los demás también lo son". El hombre no es una isla, es comunicación. Así que hay una estrecha relación entre la comunión y la búsqueda. Paulo Freire (1996, p.63).

Dada la situación por pandemia-Covid, y la obligatoriedad de la educación de mantener sus procesos de formación en el ámbito universitario, se observa que las condiciones laborales cambian abruptamente para los docentes; la readaptación a un sistema netamente en línea desde la inmovilidad del lugar de trabajo, las largas horas, el intentar resolver las situaciones de los estudiosos, responder a los sistemas administrativos universitarios, el cambio de métodos y metodologías, la responsabilidad por lograr en los estudiosos los resultados de aprendizaje, el apoyo a estudiantes que se encuentran en situaciones difíciles, el asumir situaciones del contexto, como las actividades e casa y los niños, son algunos de los factores que tienen implicación importante en la carga laboral física y mental de los docentes.

Las anteriores son razones que nos motivan a investigar sobre las percepciones y efectos en la carga laboral, física y mental a que los docentes universitarios están expuestos en este momento, de contingencia y la crisis sanitaria mundial. La presente investigación está acompańada por estudiantes de último semestre del área de salud.

La función del docente se encuentra revestida de gran importancia ya que son quienes sientan bases para el futuro desarrollo político, económico y social de las comunidades y sus países, para la UNESCO,” los docentes representan una de las fuerzas más sólidas e 
influyentes con miras a garantizar la equidad, el acceso y la calidad de la educación. Ellos son la clave del desarrollo mundial sostenible" (UNESCO, 2019, p. 2).

El análisis de la situación actual del estado de la cuestión; nos lleva a comprender que los docentes propenden por lograr aprendizajes en sus estudiosos, desarrollar distintas capacidades que formen de manera integral a la persona su pensamiento, la acción, el ser, la comunicación, la relación con el contexto y la posibilidad de aplicación de sus aprendizajes en el mundo laboral además de aportar a su desarrollo social, son otras de las labores del docente en cualquiera de las etapas de la educación.

El docente en el desarrollo de sus actividades se ve abocado a una serie de procesos que se convierten en carga laboral física, mental, emocional, familiar entre otras, todo lo anterior en situación de normalidad académica, el estilo de vida de los docentes universitarios se ve afectado por factores internos y externos diferentes del entorno laboral lo que hace necesarios abordajes en su calidad de vida desde "múltiples perspectivas, con amplitud de criterio y con la concepción sistémica y compleja de la realidad, asumiendo los archipiélagos de certeza que pudieran estar involucrados sin soslayar el océano de incertidumbres en el que navegamos, intentando configurar la red de interrelaciones de sus múltiples componentes y dimensiones" (Guevara, 2011, p. 322).

Siendo esta una problemática del mundo laboral donde está implicada la salud y bienestar, se han realizado diversos estudios sobre las percepciones del entorno laboral de los profesores universitarios, así por ejemplo, la manera en que los contextos inciden sobre las condiciones de trabajo, la calidad del mismo, su calidad de vida, salud en general, motivación en sus actividades, el compromiso, el rendimiento, eficiencia y la satisfacción de su labor, así como la presencia de disfunciones como lo son el ausentismo, accidentalidad o enfermedades laborales (Restrepo, 2016). Otra situación evidente en los docentes es el Burnout, hace referencia a las características sociodemográficas, las cuales dejan marcadores importantes en la afectación de la salud mental y física de los docentes (Marenco-Escuderos y Ávila-Toscano, 2016).

Como vemos se encuentran distintas variables y categorías implicadas en la salud mental y física de los docentes dados los contextos, factores intralaborales y extralaborales; sin embargo, por razones obvias no existen en este momento estudios que midan las percepciones sobre la incidencia de la pandemia Covid-19 en la actividad docente, por ello esta investigación lleva su foco hacia la percepción del docente universitario, ante las causas que generan carga laboral, física y mental en el proceso de contingencia por pandemia Covid-19.

Hipótesis de la investigación: Acorde a la percepción del docente universitario y dada la situación de pandemia Covid-19, existe un mayor impacto en la carga laboral físico-mental que lleva a proponer programas de salud pertinentes que aporten a aminorar este efecto. 


\section{Algunos fundamentos teóricos necesarios}

Carga laboral: Se determina que la función o la labor docente, está relacionada con procesos de enseñanza y aprendizaje, la cual debe contener métodos, didácticas, planeaciones, actualización, explicaciones, relación de procesos, evaluaciones, teniendo en cuanta los planes de estudio y los diferentes estilos de aprendizaje sin olvidar los resultados de aprendizaje propios. La función docente más que ser una función académica comprende aquellas actividades extracurriculares no lectivas, como lo es, el servicio de orientación estudiantil, atención a la comunidad estudiantil académica y a su parte representativa, la planeación de la estructuración evaluativa, toda tarea que se encuentre relacionada o estipulada con el proyecto educativo institucional, las cuales se rigen bajo la norma laboral en docencia y directivos (MEN, 2002).

La carga laboral puede llegar a afectar a la persona y una de sus manifestaciones puede ser el síndrome de Burnout (consumido o apagado) la cual genera deterioro de salud mental y física debido a la presión y al estrés laboral lo cual es factor predisponente a desarrollar enfermedades laborales como las que encontramos en la tabla de enfermedades que se encuentra en del decreto 1477 del 2014 , este síndrome va generando disminución de la calidad del trabajo del docente tomando en cuenta sus habilidades de comunicación, verbales y no verbales, su optimismo o agrado para desarrollar actividades laborales y extralaborales; se van desarrollando síntomas como dolor de cabeza, gastritis, insomnio, cambios de conducta o ansiedad, son personas que permanecen con una actitud defensiva o conductas agresivas (Olaya, 2015), afirma que este síndrome genera graves repercusiones desde el punto de vista socio-afectivos, mental y emocional en los docentes.

Carga Mental: Se relaciona con toda actividad que requiera mayor esfuerzo mental que físico, actividades con mayor manejo de la información, el trabajador se mueve en un ambiente con requerimientos mentales, cognitivos o intelectuales a lo largo de su jornada laboral, lo que aumentó el esfuerzo mental que realiza el trabajador, moviéndose así entre factores como lo son capacidad de respuesta, requisitos, contenido, así como las condiciones ambientales en las que se realiza, implica aspectos sociales, elementos psicológicos relacionados con las funciones organizativas, lo anterior debe involucrar un diseńo o rediseño del puesto de trabajo (García, 2012, p. 31). La carga mental, se asocia con tensiones que conlleva las exigencias del trabajo cognitivo; es decir: condiciones, organización y medio ambiente de trabajo relacionadas con la capacidad del trabajador para realizar su labor, que desencadena en alteraciones psicológicas o emocionales (Vilaret, 2013). Se puede comprender como relaciones e interacciones dadas por las demandas propias e la tarea en $\mathrm{n}$ términos de pensamiento y cognición, en la cuales es de vital importancia tener en cuenta las características propias de cada persona, así como su contexto y vivencias del momento. "Entre sus causas cabe citar características de la tarea, presiones temporales y ritmo de 
trabajo, así como, las funciones a desempeñar, el grado de autonomía, y la interacción con otras personas" (Ceballos, 2015, p. 20).

Carga Física: Se relaciona con toda actividad que requiera un esfuerzo físico por parte del trabajador, está compuesta por dos grupos los cuales se clasifican en dinámicos y estáticos, al hablar del grupo dinámico se deben incluir la sobrecarga generada por posturas mantenidas o estáticas, ya hablando de la sobrecarga generada de manera dinámica es dada por realización de movimientos repetitivos y manipulación manual de carga, en concordancia con lo anterior se debe valorar el riesgo de exposición a estos factores ya que pueden generar incapacidad parcial, temporal y hasta permanente (Iniesta, 2015).

\section{Objetivo general}

Analizar la percepción de Docentes de universitarios sobre la carga laboral, física y mental causada por su actividad laboral en la contingencia por pandemia Covid-19.

\section{Objetivos específicos}

Describir documentalmente la relación entre el estrés, la carga física y mental que puede que puede llegar afectar a un docente en su actividad laboral.

Analizar las características de las causas de la carga laboral, física y mental con base a las percepciones.

Relacionar algunas pautas que contribuyan a mitigar los efectos de la carga laboral, física y mental de los docentes durante la contingencia por pandemia Covid-19.

Basados en la comprensión teórica y antecedentes de la investigación, se identifican criterios para el desarrollo de las categorías de análisis, los cuales dan las pautas para el enfoque de la entrevista (Tabla 1).

Tabla 1.

Categorias de análisis

Categorías de análisis

Subcategorías de análisis

Datos personales Iniciales del nombre, edad (para la codificación), personas con las que convive y dependencia de trabajo dentro de la universidad.

Horario habitual de trabajo, actividades extralaborales, tiempo de

Contexto habitual desplazamiento de la residencia al lugar de trabajo, realización de del rol docente pausas activas y tiempo empleado para la realización de actividades laborales fuera del horario de trabajo. 


\begin{tabular}{|c|c|}
\hline $\begin{array}{l}\text { Categorías de } \\
\text { análisis }\end{array}$ & Subcategorías de análisis \\
\hline $\begin{array}{l}\text { Contexto especial } \\
\text { dado por la } \\
\text { contingencia }\end{array}$ & $\begin{array}{l}\text { Realización de pausas activas, tipo de pausa activa, tiempo de descanso } \\
\text { en el horario laboral y nivel de productividad. }\end{array}$ \\
\hline Carga laboral & $\begin{array}{l}\text { Contexto habitual: tipo de contrato laboral, funciones laborales a } \\
\text { cargo, horas laborales estipuladas por semana según contrato. } \\
\text { Contexto especial: horas reales laboradas a la semana, funciones } \\
\text { laborales adicionales. }\end{array}$ \\
\hline Carga física & $\begin{array}{l}\text { Contexto habitual: postura frecuente en el área de trabajo, descripción } \\
\text { de la silla del puesto de trabajo, cambios de posición, lugar/ } \\
\text { lugares de la universidad en los que se realiza la actividad laboral, } \\
\text { desplazamientos dentro de la jornada laboral, actividades que } \\
\text { requieran fuerza. } \\
\text { Contexto especial: lugar de la residencia donde realiza o frecuenta } \\
\text { realizar la jornada laboral, adaptación del puesto de trabajo, actividad } \\
\text { de manipulación de cargas, condiciones ambientales. }\end{array}$ \\
\hline Carga mental & $\begin{array}{l}\text { Tiempo de respuesta de trabajos por cumplimiento al cronograma } \\
\text { laboral, monotonía, cansancio durante la jornada laboral, } \\
\text { disponibilidad de tiempo realizando trabajo en línea modalidad } \\
\text { remota. }\end{array}$ \\
\hline
\end{tabular}

\section{MÉTODO}

Dada la propuesta de percepciones se hace coherente la investigación cualitativa, ya que permite un proceso interpretativo de indagación, que permite examinar un problema humano o social, construye una imagen compleja y holística (Daza, 2018), analiza palabras, así se logran obtener la perspectiva de los docentes, de forma integral, identificando la naturaleza profunda de la problemática desde una dinámica experiencial. El diseño fenomenológico, es un estudio de experiencias de vida, respecto de un suceso, ligado a la percepción, como también la profunda visualización del fenómeno, desde la construcción social y la subjetiva. "Este enfoque asume el análisis de los aspectos más complejos de la vida humana, de aquello que se encuentra más allá de lo cuantificable” (Uillen, 2019, p. 218).

\section{Participantes}

178 Docentes Universitarios del ciclo 2020-1. Horario mańana y noche, que tienen contrato laboral de medio tiempo - 4 horas diarias, o Tiempo completo 8 horas en jornada habitual, que se encuentren laborando desde sus hogares dando cátedra teórica, práctica y teórico-práctico. 


\section{Instrumentos}

Entrevista semiestructurada, que consta de 41 de preguntas mixtas abiertas y de selección múltiple, que tomó a cada docente un promedio de 5 a 10 minutos responder, dando así lugar a las percepciones que tienen los docentes ante la carga laboral, física, mental a causa de la contingencia. Para la recolección de datos y tabulación de las respuestas, se utilizó la plataforma Google Forms, de donde se obtiene resumen general de la entrevista y visibiliza las respuestas por cada participante. Procedimiento: Se realiza invitación a docentes de distintas universidades, para responder la entrevista, dado que la entrevista tenía datos de identificación solo fue respondida por 20 docentes y las respuestas no fueron reales, por lo cual se debió nuevamente realizar la entrevista omitiendo todos los datos personales, asegurando confidencialidad, y consentimiento informado, esta investigación en ningún momento hace evidencia ni de los datos de los docentes, ni de las universidades a que pertenecen, sus cargos o labores, respetando la absoluta confidencialidad dando y logrando libertad en las percepciones de los docentes. Aun así, la dificultad y preocupación de los docentes de poner en riesgo sus trabajos logramos las respuestas de 178 docentes.

\section{Análisis de datos}

Se realiza análisis descriptivo comparativo general de datos obtenidos con respecto a la habitualidad y al contexto especial dado por la contingencia para la carga laboral, física y mental. Para el análisis completo de los datos se realizaron dos matrices y una triangulación de datos (Tabla 2).

La primera matriz es de análisis cualitativo, la cual tiene como contenido: Categorías: categorías y subcategorías de la investigación. Código: en letras y número referente a cada participante. Respuestas: Generales de cada pregunta Documental: Definición bajo autor y documento. Posteriormente, se realiza triangulación de estos datos que consiste en "verificación y comparación de la información obtenida en diferentes momentos mediante distintos métodos" (Patton, 2002, pág. 67) creada en el software de creación de mapas llamado Mindomo, (Figura 1) con el fin de identificar el cruce de información obtenida y de la literatura correspondiente, para la triangulación se usaron los siguientes puntos: Categorías: Representado por un color: Subcategorías: Preguntas de cada categoría. Código: en letras y número por participante. Palabra clave: se obtuvo del cruce de información de un autor respectivo para cada categoría y de las respuestas de los docentes en la entrevista. Resultados: la presentación de los resultados de las entrevistas y su análisis se representan mediante las siguientes tablas de las cuales se deja un ejemplo. 
Tabla 2.

Primera matriz de análisis cualitativo (Ejemplo de la Matriz primer orden)

\begin{tabular}{|c|c|c|c|c|}
\hline Categoría & Códigos & Respuesta & Documental & Análisis \\
\hline $\begin{array}{l}\text { Contexto habitual } \\
\text { del rol docente } \\
\text { ¿Cuál es su } \\
\text { horario habitual } \\
\text { de trabajo? } \\
\text { ¿Qué actividades } \\
\text { realizaba fuera } \\
\text { de su jornada } \\
\text { habitual de } \\
\text { trabajo? } \\
\text { ¿Cuánto } \\
\text { tiempo tardaba } \\
\text { normalmente } \\
\text { desde su vivienda } \\
\text { al lugar de } \\
\text { trabajo? } \\
\text { En su área de } \\
\text { trabajo ¿realiza } \\
\text { pausa activa } \\
\text { diariamente? } \\
\text { ¿cuánto tiempo } \\
\text { usted utiliza para } \\
\text { la realización de } \\
\text { las actividades } \\
\text { académicas fuera } \\
\text { de su horario de } \\
\text { trabajo }\end{array}$ & $\begin{array}{l}\text { Lccl26,cecl33, } \\
\text { lbmr42, } \\
\text { kscv39, } \\
\text { Se generaron } \\
178 \text { códigos } \\
\text { según el } \\
\text { ejemplo }\end{array}$ & $\begin{array}{l}\text { 1. Tiempo completo, medio } \\
\text { tiempo } \\
\text { 2. Descanso, tareas de casa, } \\
\text { compras, ejercicio, compras, } \\
\text { pagos, actividades de desarrollo } \\
\text { humano con otra institución, } \\
\text { trabajos de mi maestría, visitas, } \\
\text { oficios de la casa, atención } \\
\text { domiciliaria, trabajo, proyectos } \\
\text { personales, cine, cenas en } \\
\text { restaurantes, cocinar junto a mi } \\
\text { esposo, tareas administrativas, } \\
3.40 \text { min desde la universidad- } \\
10 \text { minutos desde el hospital, } \\
1 \text { hora, } 2 \text { horas, } 1 \text { hora y } 30 \\
\text { minutos, } 20 \text { minutos, cuando } \\
\text { estaba sin cuarentena } 1 \text { hora } \\
\text { aproximadamente si tenía } \\
\text { que ir a la sede de Bogotá, } \\
\text { pero si me debía desplazar a la } \\
\text { sede fuera de Bogotá } 2 \text { horas } \\
\text { aproximadamente, } 3 \text { horas } \\
4 . \text { No realiza pausas activas, } \\
\text { siempre realiza pausas activas, } \\
\text { casi siempre, ocasionalmente } \\
\text { realiza pausas activas } \\
5.2-4 \text { horas al día, } 1-2 \text { horas } \\
\text { al día, más de } 4 \text { horas al día } \\
\text { ninguna. } \\
\text { Nota. Se evidencian las } \\
\text { respuestas similares. }\end{array}$ & $\begin{array}{c}\text { Contexto } \\
\text { habitual: es } \\
\text { el entorno o } \\
\text { ambiente fisco } \\
\text { en donde se } \\
\text { mueve cada } \\
\text { persona, } \\
\text { identificando } \\
\text { tiempo o } \\
\text { lugar que lo } \\
\text { rodee en el } \\
\text { momento. }\end{array}$ & $\begin{array}{l}\text { En la habitualidad del rol } \\
\text { docente se puede identificar } \\
\text { principalmente que, en su día } \\
\text { a día el tiempo se ajustaba a } \\
\text { diferentes actividades entre } \\
\text { esas el transporte desde su } \\
\text { residencia hasta su lugar de } \\
\text { trabajo, tiempo que oscila } \\
\text { entre los } 40 \text { minutos y } 2 \text { horas } \\
\text { que tenían relativamente } \\
\text { libre lo empleaban en otras } \\
\text { actividades. Se logró identificar } \\
\text { que los docentes podían } \\
\text { realizar actividades académicas } \\
\text { fuera de su horario de trabajo } \\
\text { en donde la mayoría de los } \\
\text { docentes respondió que } \\
\text { empleaban } 2-4 \text { horas al día } \\
\text { para dar cumplimiento a las } \\
\text { actividades programadas y la } \\
\text { organización personal. Los } \\
\text { participantes cuentan con } \\
\text { tiempo para realizar actividades } \\
\text { de tipo lúdico personales y con } \\
\text { sus familiares como, ejercicio, } \\
\text { salidas a centros, visitas al cine, } \\
\text { cenas dentro y fuera del hogar } \\
\text { proyectos personales, oficios } \\
\text { varios donde se concluye que } \\
\text { los docentes podían maximizar } \\
\text { su tiempo entre las actividades } \\
\text { intra y extra laborales. }\end{array}$ \\
\hline
\end{tabular}

\section{RESULTADOS}

Descripción de los resultados encontrados: Los docentes evidencian afectación en su carga física y mental debido a la contingencia por pandemia Covid-19. La comprobación de la hipótesis se da mediante el análisis realizado por la triangulación de datos (Figura 1) con el cruce de información desde la literatura y lo obtenido de la entrevista, se puede describir la relación existente de los componentes y características que atribuyen a la labor docente como lo son la jornada laboral, intensidad de trabajo, las relaciones interpersonales con respecto a los diferentes tipos de cargas desde la habitualidad al contexto especial dado por la contingencia, en donde el esfuerzo físico-mental, las situaciones de tipo estresantes en la actividad laboral y extra laboral son 
factores atribuidos al desgaste profesional del docente, la modalidad virtual ha generado que los docentes tengan conductas físicas y psicológicas cambiantes, dado que, cuando desempeñaban su jornada laboral en actividad normal, tenían rutinas diferente, podían salir de sus residencias para ir a su trabajo y desempeñarlo en su puesto-área dentro de los horarios establecidos sin límite de movilidad o desplazamiento; a causa de la contingencia estas conductas se vieron totalmente modificadas e hizo que la estructura organizacional del trabajo migrara a la virtualidad.

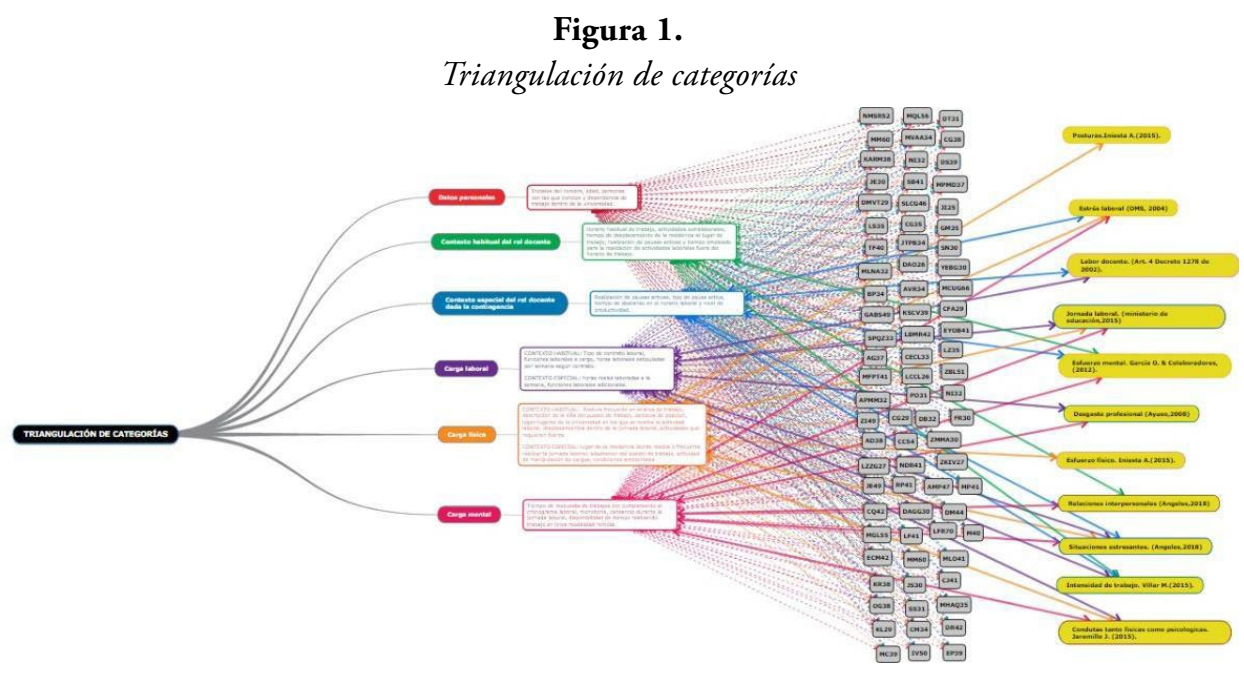

El realizar la jornada laboral dentro de sus espacios personales y familiares, limita su actividad extra laboral, esta modalidad de trabajo ha hecho que se tome tiempo adicional, restándolo al tiempo libre para cumplir actividades de tipo académico-laboral, llevando su cuerpo y mente en estado de estrés por la cantidad de trabajo existente, adicionalmente las mujeres que respondieron la entrevista, hacen referencia a la mayor carga laboral, ya que por estar en casa deben asumir tareas del hogar y actividades curriculares de sus hijos menores que también se encuentran en estudio virtual y requieren de gran ayuda, adicional a esto en algunos de los casos existen dificultades de convivencia familiar lo cual aumenta la tensión y el estrés laboral, deteriorando aún más la carga laboral y mental del docente.

\section{DISCUSIÓN Y CONCLUSIONES}

La importancia de esta investigación no solo radica en la detección de problemas serios que afectan la carga laboral y física de los docentes universitarios lo cual seguramente se extiende a todos los docentes que se encuentran en trabajo virtual y en línea, dada por la 
contingencia; sino por la predicción de problemas futuros, desencadenados de esta situación que se pueden convertir en patologías instauradas afecciones de tipo emocional y mental que requieren de trabajo continuo para restaurar el bienestar docente. Los hallazgos de esta investigación evidencian que la carga laboral en el rol docente mediante la modalidad de trabajo en casa, es notoria por diversos factores; uno de los más importantes es el horario laboral, puesto que este se aumenta en un promedio de 3 a 5 horas diarias, que en total son 15 a 25 horas semanales las cuales no son reconocidas monetariamente, al respecto Santillana en su estudio "El teletrabajo en el COVID-19, en el cual refiere que las horas laborales no tiene que cambiar fuera del estadio habitual que manejaba el trabajador y si este llega a trabajar tiempo extra tendrá que ser reconocida de forma monetaria" (Santillán, 2020 , p. 9). Otro factor importante son las tareas adicionales, como son manejo de tics innovadoras, elaboración de módulos en plataforma virtual, búsqueda de nuevos elementos para la construcción de clases diarias, siendo este factor el que más requiere tiempo y renovación en la educación universitaria. En el contexto habitual se utiliza gran tiempo en la movilización de hogar y sitio de trabajo lo cual puede considerarse como aspecto positivo en la carga laboral durante la contingencia, sin embargo, el tiempo ahorrado no mejora la carga física ni mental.

Gran parte del grupo docentes no estaban preparados para llevar su puesto de trabajo a casa lo que los obligó a realizar modificaciones con los elementos que tenían en sus hogares, aunque no fueran los adecuados, esto genero molestias e incomodidad, obligándolos a mantener posturas ergonómicamente inadecuadas, según refieren les ha empezado a producir dolores osteomusculares, por falta de tiempo tampoco se toman una pausa activa, generando desacondicionamiento físico por inactividad prolongada.

Mera (2020) en su estudio, recomendaciones prácticas para evitar el desacondicionamiento físico durante el confinamiento por pandemia asociada a COVID-19, refiere que el confinamiento en casa a generado diferentes tipos de riesgos, como los mentales (estrés, ansiedad, alteraciones de sueño) y físicos, como el desacondicionamiento, dado entre otros, por el incremento de los tiempos de inactividad física, las conductas sedentarias (Mera, Tabares, Montoya, Muñoz, y Monsalve, 2020), aunque algunos docentes toman las pausas activas esto en su mayoría sucede de forma incorrecta, ya que lo recomendable es se cambie de actividad y posición, lo cual no se está haciendo, otro factor agravante es que gran parte del grupo docentes realiza actividades extra laborales que también incluyen utilizar el computador por estudio, otros por trabajo o actividades con sus hijos, lo cual aumenta la carga física, siendo este el factor que genera más molestia e incomodidad durante el rol docente, se considera que si sigue aumentando la carga física, conduciría a la minimización de productividad del docente y así el inicio de dolores y molestias osteomusculares por exceso de trabajo y posturas erradas. Ramírez en su estudio Aspectos posturales: realiza un estudio inicial de las posturas que contribuyen al factor de riesgo por carga física en la actividad docente en el cual afirma 
que generalmente estas posturas inadecuadas y reiterativas, nos empujan hacia continuos desequilibrios estructurales que a menudo degeneran en dolencias o patologías.

La carga de trabajo físico propia de los docentes está totalmente relacionada con el trabajo de tipo cognitivo, aun así se piense que esta carga es de una sola naturaleza siempre están en relación, asumirnos entonces la postura "el esfuerzo físico y mental, una nueva mezcla peligrosa” (Ramírez, 2016, p. 136). De la misma manera los resultados de esta investigación indican que por la necesidad de realizar actividad laboral desde sus residencias su rutina cambió completamente, tanto en su tiempo laboral como extra laboral, actualmente refieren llevar una vida rutinaria, ocasionando así que exista falta de variedad en las actividades diarias, provocando un estado de aburrimiento o cansancio, así mismo Palma en su estudio De "Académicas, pandemia, encierro y bitácoras: experiencias de algunas universitarias en el contexto del COVID-19, en donde hace la aclaración del cambio de vida por la contingencia, en el cual expresa que la carga mental va a estar presente desde el momento que empieza el encierro y se aumenta la rutinariedad en el día a día, causando casi imposible no tener carga mental en esta contingencia” (Palma, 2020, p. 123), ya que eso fue una respuesta a destacar y es que bajo la modalidad virtual los docentes se sienten más cansados durante y después de su horario laboral, ya que, en la mayoría de casos, deben de emplear parte de su tiempo libre para poder dar cumplimiento al cronograma laboral que puede ser ya programado o imprevisto por el programa en algunas ocasiones; todos estos factores como lo son la falta de tiempo libre personal y para compartir con los otros, la presión laboral de cumplimiento de actividades, la monotonía en el día a día generan un esfuerzo mental deliberado que involuntariamente surge dada la necesidad de dar respuesta a las diferentes actividades laborales, adicionalmente cabe mencionar las tareas extra laborales que tienen los docentes como lo son las responsabilidades familiares, así mismo Zamarro en su estudio "Diferencias de género en el impacto de COVID-19, refiere como es esa carga mental en mujeres con trabajo en casa, teniendo a cargo el cuidado de niños por cierre de los colegios por la contingencia, por lo cual se aumenta la carga mental y se disminuye la atención laboral" (Zamarro, 2020, p. 67), tener que cumplir con sus responsabilidades del hogar también es un punto a considerar que incrementa del conjunto de tensiones inducidas en el docente por carga y esfuerzo mental que realiza para la organización personal y así mismo búsqueda de soluciones presentes en el hogar y en el trabajo, puesto que, el docente por el compromiso que tiene con su rol, no puede perder cordura ante sus actividades.

\section{REFERENCIAS}

Cárdenas, M., Méndez, L.M., y González, M.T. (2014). Evaluación del desempeño docente, estrés y burnout en profesores. Revista Electrónica "Actualidades Investigativas en Educación", 14(1), 1-22. https://www.redalyc.org/pdf/447/44729876006.pdf 
Ceballos, P. (2015). Factores psicosociales y carga mental de trabajo. Revista Latinoamericana de Enfermagem, 16, 23. https://doi.org/10.1590/0104-1169.0044.2557

Daza, W.G. (2018). Investigación educativa desde un enfoque cualitativo: la historia oral. Voces de la Educación, 3(3), 93-103. https://www.revista.vocesdelaeducacion.com. $\mathrm{mx} /$ index.php/voces/article/view/123

Freire, P. (1996). Pedagogía de la autonomía. Río de Janeiro: Paz e Terra.

García-Vega, S. (2012). Clima laboral: buscando la satisfacción de los colaboradores. Revista Análisis Laboral: Aspectos Socioeconómicos y Jurídicos, 36(421), 30-32.

Guevara, H. (2011). Calidad de vida del docente universitario vista desde la Complejidad. Revista Cubana de Salud Pública, 37(3), 314-323. http://scielo.sld.cu/scielo. php?script=sci_arttext $\&$ pid=S0864-34662011000300012

Iniesta, A. (2015). Guía sobre el manejo del estrés desde Medicina del Trabajo. Barcelona: Edición Sans Growing Brands.

Marenco-Escuderos, A.D., y Ávila-Toscano, J.H. (2016). Burnout y problemas de salud mental en docentes: diferencias según características demográficas y sociolaborales. Psychologia, 10(1), 91-100. http://www.scielo.org.co/scielo.php?pid=S190023862016000100009\&script=sci_abstract\&tlng=es

MEN (2002). DECRETO 1278 DE 2002. Bogotá- Colombia: Presidencia de la República.

Mera, A., Tabares, L., Montoya, N., Muñoz, M., y Monsalve, S. (2020). Recomendaciones prácticas para evitar el desacondicionamiento físico durante el confinamiento por pandemia asociada a COVID-19. Medellín Colombia: Universidad CES. Medellín, Colombia. Centro afiliado Cochrane Universidad del Cauca. Popayán, Colombia.

Olaya, C. (2015). Sindrome de burnout o sindrome de agotamiento profesional (SAP) en el trabajo de los docentes distritales de la localidad de USME. España: Universidad Militar Nueva Granada.

Palma, M. (2020). De académicas, pandemia, encierro y bitácoras: experiencias de algunas universitarias en el contexto del COVID-19. Costa Rica: Escuela de antropología, Universidad de Costa Rica.

Patton, M. (2002). Qualitative Evaluation and Research Methods. London: Sage.

Ramírez, P.C.E. (2016). Aspectos posturales: Estudio de las posturas que contribuyen al factor de riesgo por carga física en la actividad docente. Bogotá: Universidad ECCI.

Restrepo, J. (2016). La génesis de la Economía de la Salud en Kenneth Arrow (1963). Lecturas de Economía, (84), 209-242. http://www.scielo.org.co/scielo.php?.pid=S0120$25962016000100007 \&$ script $=$ sci_abstract $\&$ tlng=es

Santillán, W. (2020). El teletrabajo en el Covid-19. CienciAmérica, 9(2), 65-76. 
Uillen, D.E. (2019). Investigación cualitativa: Método fenomenológico hermenéutico. Propósitos y Representaciones, 7(1), $201-229$.

Vilaret, S.A., y Ortiz, I.A. (2013). Carga mental y Síndrome de Burnout en docentes a tiempo completo de una universidad de Quito. Revista EiDOS, 20, 12. https://doi. org/10.29019/eidos.v0i6.106

Zamarro, P.P. (2020). Diferencias de género en el impacto de COVID-19. California: Universidad del Sur de California.

Recibido: 20 de julio de 2020 Aceptado: 20 de agosto de 2020 